\title{
New Bounds for the $L(h, k)$ Number of Regular Grids
}

\section{Tiziana Calamoneri ${ }^{1}$, Saverio Caminiti ${ }^{1}$, Guillaume Fertin ${ }^{2}$}

\author{
1 Dipartimento di Informatica \\ Università degli Studi di Roma "La Sapienza" \\ Via Salaria 113 \\ 00198 Roma, Italy
}

\author{
2 LINA FRE CNRS 2729 \\ Université de Nantes
}

2 rue de la Houssinière, BP 92208

44322 Nantes Cedex 3, France

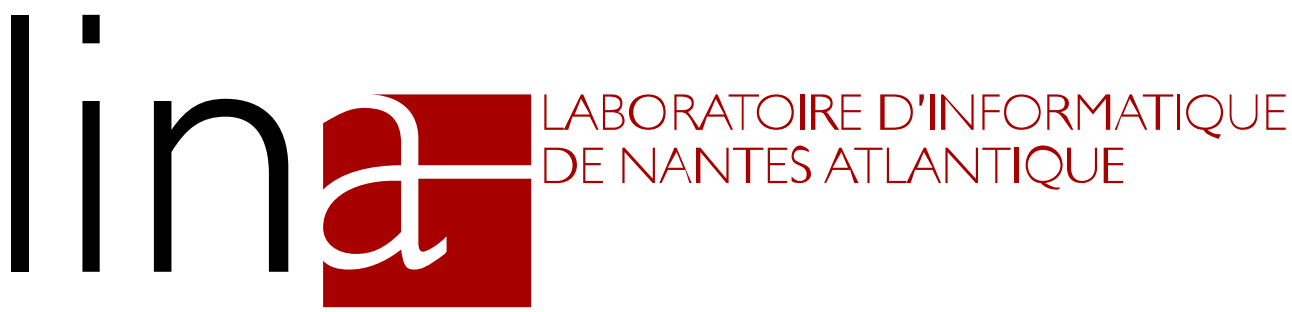

\section{RESEARCH REPORT}

\author{
$\mathbf{N}^{\mathbf{0}} 05.04$ \\ Juillet 2005
}
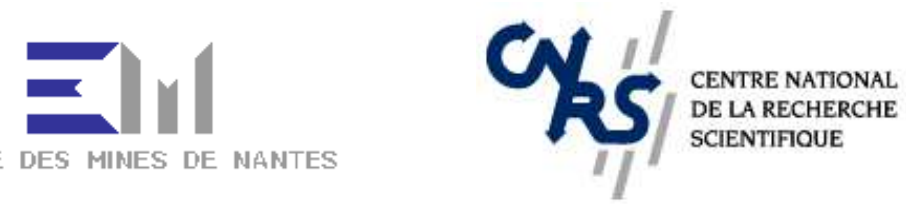

LINA, Université de Nantes - 2, rue de la Houssinière - BP 92208 - 44322 NANTES CEDEX 3

Tél. : 0251125800 - Fax. : 0251125812 - http://www.sciences.univ-nantes.fr/lina/ 
Tiziana Calamoneri, Saverio Caminiti, Guillaume Fertin

New Bounds for the $L(h, k)$ Number of Regular Grids

$18 \mathrm{p}$.

Les rapports de recherche du Laboratoire d'Informatique de Nantes-Atlantique sont disponibles aux formats PostScript ${ }^{\oplus}$ et PDF $^{\oplus}$ à l'URL :

http://www.sciences.univ-nantes.fr/lina/Vie/RR/rapports.html

Research reports from the Laboratoire d'Informatique de Nantes-Atlantique are available in PostScript ${ }^{\circledast}$ and $P D F^{\circledast}$ formats at the URL:

http://www.sciences.univ-nantes.fr/lina/Vie/RR/rapports.html

(C) July 2005 by Tiziana Calamoneri, Saverio Caminiti, Guillaume Fertin

rr0405.tex - New Bounds for the $L(h, k)$ Number of Regular Grids - 8/7/2005 - 19:08 


\title{
New Bounds for the $L(h, k)$ Number of Regular Grids
}

\author{
Tiziana Calamoneri, Saverio Caminiti, Guillaume Fertin \\ calamo@di.uniroma1.it, caminiti@di.uniroma1.it, fertin@lina.univ-nantes.fr
}

\begin{abstract}
For any non negative real values $h$ and $k$, an $L(h, k)$-labeling of a graph $G=(V, E)$ is a function $L: V \rightarrow \mathbb{R}$ such that $|L(u)-L(v)| \geq h$ if $(u, v) \in E$ and $|L(u)-L(v)| \geq k$ if there exists $w \in V$ such that $(u, w) \in E$ and $(w, v) \in E$. The span of an $L(h, k)$-labeling is the difference between the largest and the smallest value of $L$. We denote by $\lambda_{h, k}(G)$ the smallest real $\lambda$ such that graph $G$ has an $L(h, k)$-labeling of span $\lambda$. The aim of the $L(h, k)$-labeling problem is to satisfy the distance constraints using the minimum span.

In this paper, we study the $L(h, k)$-labeling problem on regular grids of degree 3, 4, 6 and 8, solving several open problems left in the literature.
\end{abstract}

Additional Key Words and Phrases: $L(h, k)$-labeling, triangular grids, hexagonal grids, squared grids, octagonal grids 



\section{Introduction}

For any non negative real values $h$ and $k$, an $L(h, k)$-labeling of a graph $G=(V, E)$ is a function $L: V \rightarrow \mathbb{R}$ such that $|L(u)-L(v)| \geq h$ if $(u, v) \in E$ and $|L(u)-L(v)| \geq k$ if there exists $w \in V$ such that $(u, w) \in E$ and $(w, v) \in E$. The span of an $L(h, k)$-labeling is the difference between the largest and the smallest value of $L$. Hence, it is not restrictive to assume 0 as the smallest value of $L$, something which will be assumed throughout this paper. We denote by $\lambda_{h, k}(G)$ the smallest real $\lambda$ such that graph $G$ has an $L(h, k)$-labeling of span $\lambda$; we call $L(h, k)$ number of $G$ this value. The aim of the $L(h, k)$-labeling problem is to satisfy the distance constraints using the minimum span.

Since its definition [11] as a specialization of the frequency assignment problem in wireless networks [12, 16], the $L(h, k)$-labeling problem has been intensively studied. Note that the $L(h, k)$-labeling problem is a generalization of some standard graph colorings, such as the usual (or proper) coloring when $k=0$, or the 2-distance coloring (equivalent to the proper coloring of the square of the graph) when $h=k$. We also note that the case $h=2$ and $k=1$ (or, more generally $h=2 k$ ), called radio-coloring or $\lambda$-coloring, is the most widely studied (see for instance $[7,9,13,14])$.

The decision version of the $L(h, k)$-labeling problem is NP-complete even for small values of $h$ and $k$ [2]. This motivates seeking optimal solutions on particular classes of graphs (see for instance $[3,4,8,11,17,18$, 19] and [6] for a complete survey). Concerning the more specific grid topologies, a large number of papers has been published on the subject. For instance, Makansi [15] provided an optimal $L(0,1)$-labeling for squared grids. Battiti, Bertossi and Bonuccelli [1] found an optimal $L(1,1)$-labeling for hexagonal, squared and triangular grids. The $L(2,1)$-labeling problem of regular grids of degree $\Delta$, denoted $G_{\Delta}$, has been studied independently by different authors [3, 7] proving that $\lambda_{2,1}\left(G_{\Delta}\right)=\Delta+2$ by means of optimal coloring algorithms. More recently, Fertin and Raspaud [10] determined several bounds on $\lambda_{h, k}$ for $d$-dimensional squared grids.

In [5] some values of $\lambda_{h, k}$ for regular grids of degree 3,4 , and 6 are exactly computed, while in some intervals different upper and lower bounds are given ; the case $h<k$ is not considered at all.

In this paper, we study the $L(h, k)$-labeling problem on regular grids of degree 3,4 , and 6 for those values of $h$ and $k$ whose $\lambda_{h, k}$ is either not known or not tight. Moreover, for the first time in the literature, we investigate on the problem for grids of degree 8 . For all considered grids, in some cases we provide exact results, while in the other ones we give very close upper and lower bounds. A graphical representation of the four types of grids studied in this paper is given in Figure 1, while a summary of our results is depicted in Figure 2.

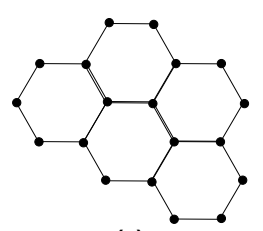

(a)

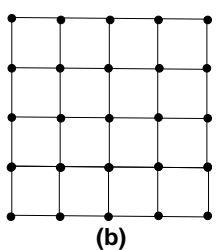

(b)

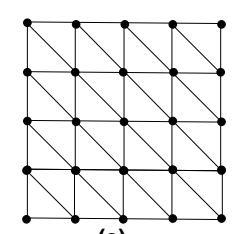

(c)

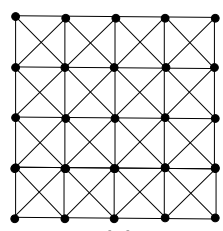

(d)

Figure 1: Grids studied in this paper: (a) $G_{3}$, (b) $G_{4}$, (c) $G_{6}$ and (d) $G_{8}$

\section{Preliminaries}

In this section, we show four different lemmas, which will prove to be useful in the rest of the paper. Lemmas 1 and 2 are concerned with lower bounds for the $L(h, k)$ number, while Lemmas 3 and 4 deal with upper bounds.

Lemma $1 \lambda_{h, k}\left(G_{\Delta}\right) \geq h+(\Delta-1) k$ when $h \leq k$, for $\Delta=3,4$.

Proof : Consider an optimal $L(h, k)$-labeling of $G_{\Delta}, h \leq k, \Delta=3,4$, and let $x$ be a node labeled 0 . The smallest label among those of their neighbors must be at least $h$. Furthermore, the $\Delta$ neighbors of $x$ are all connected by a 2-length path and hence their labels must differ at least $k$ from each other. It follows that the greatest label must be at least $h+(\Delta-1) k$. 


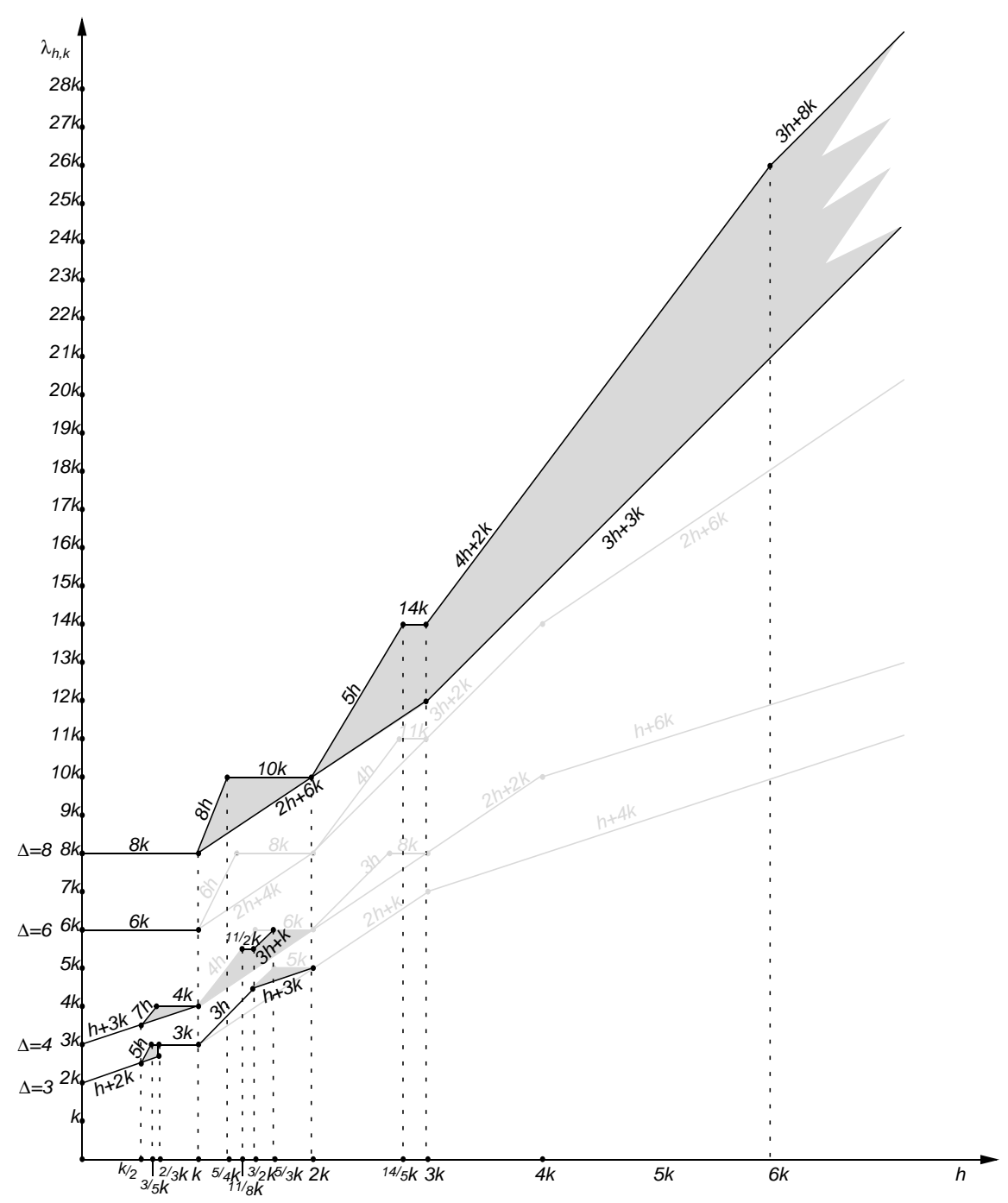

Figure 2: Summary of the results achieved in this paper: bold lines are results from this paper, while gray lines are previously known lower and upper bounds.

Lemma $2 \lambda_{h, k}\left(G_{\Delta}\right) \geq \Delta k$ when $h \leq k$, for $\Delta=6,8$.

Proof : Observe that $G_{6}$ and $G_{8}$ are characterized by the property that each pair of adjacent nodes is also connected by a 2-length path. This implies that, given an optimal $L(h, k)$-labeling of $G_{\Delta}, h \leq k, \Delta=6,8$, starting from a node $x$ labeled 0 , the smallest label, among those of their neighbors must be at least $k$. With reasonings analogous to those of the previous proof, the claim follows.

Lemma 3 For any graph $G$ and any $0 \leq h \leq k, \lambda_{h, k}(G) \leq k \cdot \lambda_{1,1}(G)$.

Proof : Consider an optimal $L(1,1)$-labeling, say $\mathcal{L}$, of $G$. Consider the labeling $\mathcal{L}^{\prime}$ obtained from $\mathcal{L}$ by substituting every label $i$ with label $i k\left(i=0,1, \ldots, \lambda_{1,1}(G)\right)$. We claim that $\mathcal{L}^{\prime}$ is an $L(h, k)$-labeling of $G$ with span $k \cdot \lambda_{1,1}(G)$, provided $h \leq k$. Indeed, any two neighbors, which differ by at least 1 in $\mathcal{L}$, differ by at least $k \geq h$ in $\mathcal{L}^{\prime} ;$ moreover, any two nodes connected by a 2-length path, which differ by at least 2 in $\mathcal{L}$ differ by at least $2 k \geq k$ in $\mathcal{L}^{\prime}$.

Lemma 4 For any graph $G$ and any $h \geq \frac{k}{2}, \lambda_{h, k}(G) \leq h \cdot \lambda_{1,2}(G)$. 
Proof : Analogously to proof of Lemma 3, consider an $L(1,2)$ labeling, say $\mathcal{L}$, of $G$. Consider the labeling $\mathcal{L}^{\prime}$ obtained from $\mathcal{L}$ by substituting every label $i$ with label $i h\left(i=0,1, \ldots, \lambda_{1,2}(G)\right)$. Since $h \geq \frac{k}{2}, \mathcal{L}^{\prime}$ is an $L(h, k)$ labeling of $G$ with span $h \cdot \lambda_{1,2}(G)$. Indeed, any two neighbors, which differ by at least 1 in $\mathcal{L}$, differ by at least $h$ in $\mathcal{L}^{\prime}$; moreover, any two nodes connected by a 2-length path, which differ by at least 2 in $\mathcal{L}$ differ by at least $2 h \geq k$ in $\mathcal{L}^{\prime}$.

If no confusion arises, we will speak interchangeably, in the rest of this paper, of a node and its label.

\section{Regular Grids of Degree 3}

\subsection{Upper Bounds}

Proposition $1 \lambda_{h, k}\left(G_{3}\right) \leq h+2 k$ when $h \leq \frac{k}{2}$.

Proof : Consider an optimal $L(1,2)$-labeling of $G_{3}$ over the set of colors $\{0,1, \ldots, 5\}$, as shown in Figure 3(a). The idea is to substitute $h$ to $1, k$ to $2, h+k$ to $3,2 k$ to 4 , and $h+2 k$ to 5 . In that case, the labeling that is produced is a feasible $L(h, k)$-labeling. Indeed, each pair of consecutive labels differ by either $h$ or $k-h$, but since we supposed $h \leq \frac{k}{2}$, we have $k-h \geq h$ and thus any two consecutive labels differ by at least $h$. Similarly, any other pair of distinct labels differ by at least $k$. Moreover, the largest label used is $h+2 k$, hence the result.

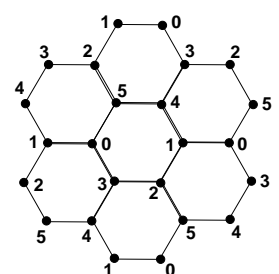

(a)

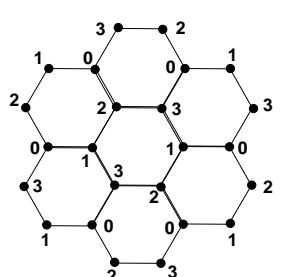

(b)

Figure 3: $L(h, k)$-labeling of $G_{3}$ : (a) $L(1,2)$-labeling ; (b) $L(1,1)$-labeling

Proposition $2 \lambda_{h, k}\left(G_{3}\right) \leq \min \{5 h, 3 k\}$ when $\frac{k}{2} \leq h \leq k$.

Proof : By Lemma 4, since $\frac{k}{2} \leq h$ and since there exists an $L(1,2)$-labeling of $G_{3}$ that is of span 5 (as shown in Figure 3(a)), we know there exists an $L(h, k)$-labeling of $G_{3}$ of span $5 h$.

Analogously, since $h \leq k$, we obtain an $L(h, k)$-labeling of span $3 k$ by Lemma 3 ; indeed, there exists an $L(1,1)$ labeling of $G_{3}$ that is of span 3 (as shown in Figure 3(b)).

\subsection{Lower Bounds}

Proposition $3 \lambda_{h, k}\left(G_{3}\right) \geq h+2 k$ when $h \leq k$.

Proof : This bound directly comes from Lemma 1.

Proposition $4 \lambda_{h, k}\left(G_{3}\right) \geq 3 k$ when $\frac{2}{3} k \leq h \leq k$.

Proof : Consider an optimal $L(h, k)$-labeling of $G_{3}$. Suppose, by contradiction, that $\lambda_{h, k}\left(G_{3}\right)<3 k$. Let us consider a node labeled 0 , and let $x, y$, and $z$ be its 3 neighbors. Without loss of generality, suppose $x<y<z$. In view of the $L(h, k)$-constraints, we must have $x \geq h, y \geq x+k \geq h+k$, and $z \geq y+k \geq h+2 k$. Furthermore, from the hypothesis $\lambda_{h, k}\left(G_{3}\right)<3 k$, we have that $z<3 k$, hence $y \leq z-k<2 k$, and $x \leq y-k<k$. Let $x_{1}$ and $x_{2}, y_{1}$ and $y_{2}, z_{1}$ and $z_{2}$ be the not 0 neighbors of $x, y$, and $z$, respectively (see Figure 4).

Let us first prove that if $y_{m}=\min \left\{y_{1}, y_{2}\right\}$ and $y_{M}=\max \left\{y_{1}, y_{2}\right\}$, then $y_{m}<y<y_{M}$. Indeed, if $y<y_{m}$, then $y_{m} \geq y+h \geq 2 h+k$, and consequently $y_{M} \geq 2 h+2 k$. However, $2 h+2 k \geq 3 k$ (because we supposed $h \leq \frac{2 k}{3} \geq \frac{k}{2}$ ), a contradiction to the fact that $\lambda<3 k$. On the other hand, if $y_{M}<y$, then $y \geq y_{M}+h$. And 


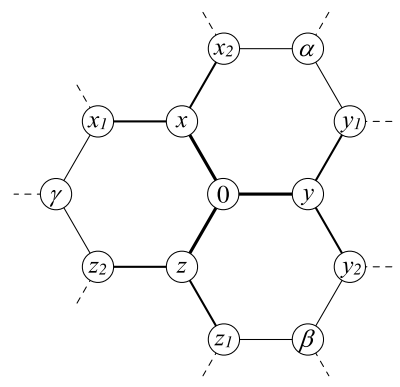

Figure 4: Neighborhood of a node labeled 0 in $G_{3}$

since $y_{M} \geq y_{m}+k \geq 2 k$, we end up with $y \geq h+2 k$. However, by hypothesis we know that $y<2 k$, a contradiction since $3 h-k \leq h+2 k$, because we supposed $h \leq \frac{3 k}{2}$. Thus we conclude that in all the cases, we have $y_{m}<y<y_{M}$.

Now, in order to prove the statement, we will show that under the hypothesis $\lambda_{h, k}\left(G_{3}\right)<3 k$, both cases $x_{1}<x_{2}$ and $x_{1}>x_{2}$ lead to a contradiction.

Case 1: $x_{1}<x_{2}$. This implies $x_{1} \geq k$, as $x_{1}$ is connected by a 2-length path to node 0 (via $x$ ) and $x_{2} \geq$ $x_{1}+k \geq 2 k$. If $x_{1}<x$, then $x \geq x_{1}+h \geq k+h$, a contradiction since $x<k$. Hence, $x<x_{1}<x_{2}$. It follows that $x_{1} \geq x+h \geq 2 h$ and $x_{2} \geq x_{1}+k \geq 2 h+k$. Let us now consider $y_{1}$ and $y_{2}$.

Case 1.1: $y_{1}<y_{2}$. Hence we know that $y_{1}<y<y_{2}$. In such a case $y_{1} \geq k$ and $y_{1} \leq y-h<2 k-h$. Note that $y_{1}<x_{2}$ as $y_{1}<2 k-h$ and $x_{2} \geq 2 k$. Let us consider the common neighbor of $x_{2}$ and $y_{1}, \alpha$, and let us study the relative position of its label with respect to $x_{2}$ and $y_{1}$.

- $\alpha<y_{1}<x_{2}$. Then $\alpha \leq y-k<k$ : if $x<\alpha$ we have $\alpha \geq x+k \geq h+k$, a contradiction; on the other hand, if $\alpha<x$ then $\alpha \leq x-k<0$, a contradiction too.

- $y_{1}<x_{2}<\alpha$. Then $x_{2} \leq \alpha-h<3 k-h$; from previous hypotheses we also have $x_{2} \geq 2 h+k$, and this leads to a contradiction as $3 k-h \leq 2 h+k$ when $h \geq \frac{2}{3} k$.

- $y_{1}<\alpha<x_{2}$. We have again two cases. If $y_{1}<\alpha<y$ then $\alpha \leq y-k<k$ and $y_{1} \leq \alpha-h<k-h$ that is a contradiction as $y_{1} \geq k$. If $y_{1}<y<\alpha$ then $\alpha \leq x_{2}-h \leq 3 k-h, y \leq \alpha-k<2 k-h$, and $y_{1} \leq y-h<2 k-2 h$ that is a contradiction as $y_{1} \geq k$ and $k<2 k-2 h$ when $h>\frac{2}{3} k$.

Case 1.2: $y_{1}>y_{2}$. Thus we have $y_{1}>y>y_{2}$. This implies that $y_{1} \geq y+h \geq 2 h+k$. Hence, $y_{1}$ lies in the interval $\left[2 h+k ; 3 k\left[\right.\right.$. However, we also know that $x_{2}$ lies in the interval $[2 h+k ; 3 k[$. Since this interval is of width $w<2 k-2 h$, we conclude that $w<k$ (because we supposed $h \geq \frac{2 k}{3}$ and hence $h \geq \frac{k}{2}$ ). This leads to a contradiction because $y_{1}$ and $x_{2}$ must be at least $k$ away from each other.

Case 2: $x_{1}>x_{2}$. With considerations analogous to those done for case $x_{1}<x_{2}$, we can derive $x<x_{2}<x_{1}$ and $2 h+k \leq x_{1}<3 k$ and $2 h \leq x_{2}<2 k$. Now, let us look at $y_{1}$ and $y_{2}$.

Case 2.1: $y_{1}<y_{2}$. We thus have $y_{1}<y<y_{2}$. However, this leads to a contradiction. Indeed, $y_{1}>k$ as it is connected by a 2-length path to node 0 , then $x_{2} \geq y_{1}+k>2 k$ and $x_{1} \geq x_{2}+k>3 k$.

Case 2.2: $y_{1}>y_{2}$. We then have $y_{2}<y<y_{1}$. This implies that $y_{1} \geq y+h \geq 2 h+k$ and hence $y_{1}<x_{2}$ as $x_{2}<2 k$. Now consider $\alpha$, the common neighbor of $x_{2}$ and $y_{1}$.

- $x_{2}<y_{1}<\alpha$. Then $\alpha \geq y_{1}+h \geq 3 h+k \geq 3 k$, a contradiction since we supposed $\lambda<3 k$.

- $\alpha<x_{2}<y_{1}$. Then $\alpha \leq x_{2}-h<2 k-h$. If $\alpha>y$ then $\alpha \geq y+k \geq h+2 k$ that is absurd ; if $\alpha<y$ then $\alpha \leq y-k \leq k$. However, we know that $x<k$; moreover, because $\alpha<k$ and $\alpha$ must lie at least $k$ away from $x$, this leads to a contradiction. 
- $x_{2}<\alpha<y_{1}$. Then $\alpha \leq y_{1}-h<3 k-h$. If $\alpha>y$ then $\alpha \geq y+k \geq h+2 k$ that is greater than $3 k-h$ under the hypothesis $h \geq \frac{2}{3} k$; if $\alpha<y$ then $\alpha \leq y-k \leq k$ that again contradicts the fact that $\alpha$ must lie at least $k$ away from $x$.

Altogether, we see that every possible case leads to a contradiction. This proves that the initial assumption, $\lambda<3 k$, is false, and consequently the proposition is proved.

Proposition $5 \lambda_{h, k}\left(G_{3}\right) \geq 3 h$ when $k \leq h \leq \frac{3}{2} k$.

Proof: The proof is analogous to the previous one, i.e. by contradiction we assume that there exists a $L(h, k)$ labeling with span $\lambda<3 h$, we start from node labeled 0 , we look at its neighbors and prove that neither $x_{1}<x_{2}$ nor $x_{1}>x_{2}$ can occur. Wlog, let us assume $x<y<z$. Hence, $x \geq h, y \geq h+k$ and $z \geq h+2 k$. From the other hand, $z<3 h, y<3 h-k$ and $x<3 h-2 k$. Let $x_{1}$ and $x_{2}, y_{1}$ and $y_{2}, z_{1}$ and $z_{2}$ be the not 0 neighbors of $x, y$, and $z$, respectively (see Figure 4 ).

We first prove that if $y_{m}=\min \left\{y_{1}, y_{2}\right\}$ and $y_{M}=\max \left\{y_{1}, y_{2}\right\}$, then $y_{m}<y<y_{M}$. Indeed, if $y<y_{m}$, then $y_{m} \geq y+h \geq 2 h+k$, and consequently $y_{M} \geq 2 h+2 k$. However, $2 h+2 k \geq 3 h$ (because we supposed $\left.h \leq \frac{3 k}{2}\right)$, a contradiction to the fact that $\lambda<3 h$. On the other hand, if $y_{M}<y$, then $y \geq y_{M}+h$. And since $y_{M} \geq y_{m}+k \geq 2 k$, we end up with $y \geq h+2 k$. However, by hypothesis we know that $y<3 h-k$, a contradiction since $3 h-k \leq h+2 k$, because we supposed $h \leq \frac{3 k}{2}$. Thus we conclude that in all the cases, we have $y_{m}<y<y_{M}$. Now, as in the previous proof, let us consider $x_{1}$ and $x_{2}$ (see Figure 4), and show that, under the hypothesis $\lambda<3 h$, none of the cases $x_{1}<x_{2}$ and $x_{1}>x_{2}$ can occur.

Case 1: $x_{1}<x_{2}$. This implies $x_{1} \geq k$, as $x_{1}$ is connected by a 2-length path to node 0 (via $x$ ). If $x_{1}<x$, then $x \geq x_{1}+h \geq h+k$, that is a contradiction as $x<3 h-2 k \leq h+k$ under the hypothesis $h \leq \frac{3}{2} k$. Hence, $x<x_{1}<x_{2}$. It follows that $x_{1} \geq x+h \geq 2 h$ and $x_{2} \geq x_{1}+k \geq 2 h+k$. Let us consider now $y_{1}$ and $y_{2}$.

Case 1.1: $y_{1}<y_{2}$. Then we know that $y_{1}<y<y_{2}$. Note that $y_{1}<x_{2}$ as $x_{2} \geq 2 h+k$ and $y_{1} \leq y-h \leq$ $y_{2}-2 h<3 h-2 h=h$. Now, let us consider $\alpha$, common neighbor of $y_{1}$ and $x_{2}$.

- $y_{1}<x_{2}<\alpha$. The contradiction comes from the inequality $\alpha \geq x_{2}+h \geq 3 h+k$.

- $\alpha<y_{1}<x_{2}$. Then $y_{1} \geq \alpha+h \geq h, y \geq y_{1}+h \geq 2 h$ and $y_{2} \geq y+h \geq 3 h$, a contradiction.

- $y_{1}<\alpha<x_{2}$. Since we have $y_{1} \geq k$, this implies $\alpha \geq y_{1}+h \geq h+k$ and $\alpha \leq x_{2}-h<2 h$. It is easy to see that the same bounds hold also for $y$. Hence $y$ and $\alpha$ both lie in the interval $[h+k ; 2 h[$, of width $w<h-k$, that is $w \leq k$. The contradiction comes from the fact that $\alpha$ and $y$ being connected by a 2-length path, they must lie at least $k$ away from each other.

Case 1.2: $y_{1}>y_{2}$. Thus, we know that $y_{1}>y>y_{2}$. We know that $x_{2}$ and $y_{1}$ must be at least $k$ away from each other. Moreover, $2 h+k \leq x_{2}<3 h$ and $2 h+k \leq y_{1}<3 h$. Hence, both $x_{2}$ and $y_{1}$ lie in an interval of width $w<h-k$. Since we supposed $h \leq \frac{3 k}{2}$, we conclude $w<k$, a contradiction.

Case 2: $x_{1}>x_{2}$. We can easily see that in that case we must have $x_{1}>x_{2}>x$. Indeed, $x_{2} \geq k$, since it is connected by a 2-length path to node 0 . Hence, if $x>x_{2}$, then $x \geq h+k$. However, we know that $x<3 h-2 k$, a contradiction since $h \leq \frac{3 k}{2}$. Hence we conclude that $x_{1}>x_{2}>x$, which implies $x_{2} \geq x+h \geq 2 h$ and $x_{1} \geq x_{2}+k \geq 2 h+k$. Now let us consider $y_{1}$ and $y_{2}$.

Case 2.1: $y_{1}>y_{2}$. Let us then consider $\alpha$, the common neighbor of $y_{1}$ and $x_{2}$, and let us look at its relative position compared to $x$ and $y$. There are three possible cases.

- $\alpha>y>x$. We recall that we are in the case $x_{1}>x_{2}>x$, that is $x_{2} \geq x+h \geq 2 h$. If $\alpha>x_{2}$ then $\alpha \geq x_{2}+h \geq 3 h$, a contradiction to the hypothesis $\lambda<3 h$. Now, if $\alpha<x_{2}, \alpha \leq x_{2}-h$. Since $x_{2} \leq x_{1}-k<3 h-k$, we conclude $\alpha \leq 2 h-k$. But $y \geq h+k$ and $\alpha \geq y+k$, that is $\alpha \geq h+2 k$. This is a contradiction since $2 h-k \leq h+2 k$, by the hypothesis that $h \leq \frac{3 k}{2}$.

- $y>\alpha>x$. We then conclude that $\alpha \leq y-k<3 h-2 k$. On the other hand, we have $\alpha \geq x+k \geq h+k$. This is a contradiction since $h+k \geq 3 h-2 k$ due to the fact that we supposed $h \leq \frac{3 k}{2}$. 
- $y>x>\alpha$. In that case, if $\alpha<y_{1}$, then $y_{1} \geq \alpha+h \geq h$, which implies $y \geq 2 h$ and $y_{2} \geq 3 h$, a contradiction to the hypothesis $\lambda<3 h$. Now, if $\alpha>y_{1}$, then $\alpha \geq h$, which in turns means that $x \geq h+k$ and $y \geq h+2 k$. However, we know that $y<3 h-k$, a contradiction since $3 h-k \leq h+2 k$ due to the fact that we supposed $h \leq \frac{3 k}{2}$.

Case 2.2: $y_{1}>y_{2}$. Here, we consider the three nodes $z, z_{1}$ and $z_{2}$. We first show that if $z_{m}=\min \left\{z_{1}, z_{2}\right\}$ and $z_{M}=\max \left\{z_{1}, z_{2}\right\}$, then $z_{m}<z_{M}<z$. Indeed, if $z_{M}>z$ then $z_{M} \geq z+h$, and since we know $z \geq h+2 k$, we conclude $z_{M} \geq 2 h+2 k$, a contradiction to the fact that $\lambda<3 h$ since $2 h+2 k \geq 3 h$. Now let us look at the relative positions of $z_{1}$ and $z_{2}$. There are two cases to consider.

- $z_{1}>z_{2}$. In that case, we have $z>z_{1}>z_{2}$. Now let us look at $\beta$, common neighbor of $z_{1}$ and $y_{2}$, and let us consider the relative positions of $\beta$ and $y$.

$-\beta<y$. First, we note that $\beta<z_{1}$. Indeed, $z_{2} \geq k$ (it is connected by a 2 -length path to node 0 ), thus $z_{1} \geq 2 k$. However, $\beta<y$ by hypothesis, hence $\beta \leq y-k$, that is $\beta<2 h-k$. Moreover, $2 h-k \leq 2 k$ since we are in the case $h \leq \frac{3 k}{2}$, and thus we conclude that $\beta<z_{1}$. This implies $\beta \leq z_{1}-h$, that is $\beta \leq z-2 h$; and since $z \leq \lambda<3 h$, we get $\beta<h$. On the other hand, $y_{2}<y$, thus $y_{2} \leq y-h$. But since $y<2 h$, we then have $y_{2}<h$. Hence, both $\beta$ and $y_{2}$ lie in the interval $[0 ; h[$. However, they are neighbors and thus should have labels that are at least $h$ away, a contradiction.

$-\beta>y$. Then we have $\beta \geq y+k$, that is $\beta \geq h+2 k$. However, we know that $z \geq h+2 k$ as well. Thus, $\beta$ and $z$ lie in the interval $[h+2 k ; \lambda$, where $\lambda<3 h$ by hypothesis. Thus the width of this interval $w$ satisfies $w<2 h-2 k$, and thus $w<k$ because we supposed $h \leq \frac{3 k}{2}$. However, $\beta$ and $z$ are neighbors, and thus should have labels at least differing by $h$, a contradiction with the fact that $w<h$.

- $z_{2}>z_{1}$. In that case, we know that $z>z_{2}>z_{1}$. In particular, this means that $z_{2}<2 h$, and $z_{1}<2 h-k$. However, $z_{1} \geq k$ since it is connected by a 2-length path to node 0 . We also have $y \leq z-h<2 h$, and thus $y_{2} \leq y-h<h$; and since $h \geq k$, we conclude that $y_{2} \leq 2 h-k$. Moreover, $y_{2} \geq k$ since it is connected by a 2-length path to node 0 . Hence, both $z_{1}$ and $y_{2}$ lie in the interval $[0 ; 2 h-k$, of width $w<2 h-2 k$, that is $w<k$ since we supposed $h \leq \frac{3 k}{2}$. However, $z_{1}$ and $y_{2}$ are connected by a 2-length path, and thus should have labels at least differing from $k$, a contradiction.

Altogether, we see that every possible case leads to a contradiction. This proves that the initial assumption, $\lambda<3 h$, is false, and consequently the proposition is proved.

Proposition $6 \lambda_{h, k}\left(G_{3}\right) \geq h+3 k$ when $\frac{3}{2} k \leq h \leq 2 k$.

Proof : Consider an optimal $L(h, k)$-labeling of $G_{3}$ with span $\lambda$. By contradiction, suppose $\lambda<h+3 k$. Let us consider a node labeled 0 , and let $x, y$, and $z$ be its 3 neighbors. Without loss of generality, suppose $x<y<z$. In view of the $L(h, k)$-constraints, we must have $x \geq h, y \geq x+k \geq h+k$, and $z \geq y+k \geq h+2 k$. Furthermore, for the hypothesis $\lambda<h+3 k, z<h+3 k$, hence $y \leq z-k<h+2 k$, and $x \leq y-k<h+k$. Let $x_{1}$ and $x_{2}$, $y_{1}$ and $y_{2}, z_{1}$ and $z_{2}$ be the not 0 neighbors of $x, y$, and $z$, respectively (see Figure 4 ).

Let us first prove the following, which will be useful in the rest of the proof: if $y_{m}=\min \left\{y_{1}, y_{2}\right\}$ and $y_{M}=$ $\max \left\{y_{1}, y_{2}\right\}$, then $y_{m}<y<y_{M}$. Indeed, if $y<y_{m}<y_{M}$, we have $y_{m} \geq y+h \geq 2 h+k$, and $y_{M} \geq y_{m}+k \geq 2 h+2 k$. However, this contradicts the fact that $\lambda<h+3 k$, because $2 h+2 k \geq h+3 k$ (since we supposed $h \geq \frac{3 k}{2}$ ). Now suppose $y_{m}<y_{M}<y$. Then $y_{m} \geq k$, because it is connected by a 2-length path to node 0 . Thus $y_{M} \geq y_{m}+k \geq 2 k$, and $y \geq y_{M}+h \geq h+2 k$, which contradicts the fact that $y<h+2 k$. Altogether, we conclude that the only possible case is $y_{m}<y<y_{M}$ (1).

In the following we show that, under the hypothesis $\lambda<h+3 k$, both cases $x_{1}<x_{2}$ and $x_{1}>x_{2}$ lead to a contradiction, which will prove the statement.

Case 1: $x_{1}<x_{2}$. This implies $x_{1} \geq k$, as $x_{1}$ is connected by a 2-length path to node 0 (via $x$ ) and $x_{2} \geq$ $x_{1}+k \geq 2 k$. If $x_{1}<x$, then $x \geq x_{1}+h \geq k+h$, that is a contradiction as $x<k$. Hence, we have $x<x_{1}<x_{2}$. It follows that $x_{1} \geq x+h \geq 2 h$ and $x_{2} \geq x_{1}+k \geq 2 h+k$. Moreover, $x_{1} \leq x_{2}-k<h+2 k$ and $x \leq x_{1}-h<2 k$. Let us now consider $y_{1}$ and $y_{2}$. 
Case 1.1: $y_{1}<y_{2}$. By (1) above, we have $y_{1}<y<y_{2}$. Let us now consider $\alpha$ (common neighbor of $y_{1}$ and $x_{2}$ ), and let us study its relative position compared to $x$ and $y$ (we recall that $x<y$ by hypothesis).

- $\alpha>y>x$. Hence we have $\alpha \geq y+k \geq h+2 k$. But $x_{2} \geq 2 h+h \geq h+2 k$ as well. Hence, both $\alpha$ and $x_{2}$ lie in the interval $\left[h+2 k ; h+3 k\left[\right.\right.$, of width $w<k \leq h$. However, $x_{2}$ and $\alpha$ are neighbors, thus they must be at least $h$ away, a contradiction.

- $y>\alpha>x$. In that case, $\alpha \leq y-k<2 k$. But we also have $\alpha \geq x+k \geq h+k$, a contradiction.

- $y>x>\alpha$. Since $x<2 k$, we conclude that $\alpha \leq x-k<k$. However, we know $y_{1} \geq k$ (because it is connected by a 2-length path to node 0 ). Thus $\alpha<y_{1}$, hence $y_{1} \geq \alpha+h \geq h$. But we know $y_{1}<y<y_{2}$, thus $y_{1} \leq y-h$, and $y \leq y_{2}-h<3 k$, thus $y_{1}<3 k-h$. But we cannot have $y_{1} \geq h$ and $y_{1}<3 k-h$, since $h \geq \frac{3 k}{2}$.

Case 1.2: $y_{2}<y_{1}$. By (1) above, we have $y_{2}<y<y_{1}$. Hence $y_{1} \geq y+h \geq 2 h+k$. We also know that $x_{2} \geq 2 h+k$, since $x<x_{1}<x_{2}$. Thus $y_{1}$ and $x_{2}$ share the same interval [ $2 h+k ; h+3 k$ [, of width $w<2 k-h \leq k$. But $y_{1}$ and $x_{2}$ are connected by a 2-length path, and thus must be at least $k$ away, which is impossible.

Hence, at this point we conclude that necessarily $x_{1}>x_{2}$. Thus let us consider this case.

Case 2: $x_{2}<x_{1}$. In that case, it is easily seen that actually $x_{1}>x_{2}>x$, since $x>x_{2}$ would imply $x \geq x_{2}+h$; and since $x_{2} \geq k$ (it is connected by a 2-length path to node 0 ), we would have $x \geq h+k$, a contradiction to the fact that $x<h+k$. Now let us look again at the relative positions of $y_{1}$ and $y_{2}$.

Case 2.1: $y_{1}<y_{2}$. By (1) above, we have $y_{1}<y<y_{2}$. This implies that $y \leq y_{2}-h<3 k$. And since we know by hypothesis that $x<y$, we conclude that $x \leq y-k<2 k$.

- $\alpha>y>x$. Then $\alpha \leq x-k<k$. However, $y_{1} \geq k$ (it is connected by a 2-length path to node 0 ). Thus $y_{1}>\alpha$, which means $y_{1} \geq \alpha+h \geq h$. But we know that $y_{1}<y$, that is $y_{1} \leq y-h<3 k-h$. This is a contradiction since $h \geq 3 k-h$ by hypothesis.

- $y>\alpha>x$. Then $\alpha \geq x+k \geq h+k$, and $\alpha \leq y-k<2 k$. This is a contradiction since $h+k \geq 2 k$ by hypothesis.

- $y>x>\alpha$. Then $\alpha \geq y+k \geq h+2 k$. However, we know $x_{2}<x_{1}$, that is $x_{2} \leq x_{1}-k<h+2 k$, hence we conclude $\alpha>x_{2}$. Thus $\alpha \geq x_{2}+h$, and since $x_{2}>x$ we have $x_{2} \geq x+h \geq 2 h$, we conclude $\alpha \geq 3 h$, a contradiction to the fact that $\lambda<h+3 k$, since we supposed $h \geq \frac{3 k}{2}$.

Case 2.2: $y_{1}>y_{2}$. By (1) above, we have $y_{2}<y<y_{1}$. Let us now look at the relative positions of $z, z_{1}$ and $z_{2}$. We first note that if $z_{m}=\min \left\{z_{1}, z_{2}\right\}$ and $z_{M}=\max \left\{z_{1}, z_{2}\right\}$, then $z_{m}<z_{M}<z$. Indeed, if $z_{M}>z$ then $z_{M} \geq z+h$, and since we know $z \geq h+2 k$, we conclude $z_{M} \geq h+3 k$, a contradiction.

- $z_{1}>z_{2}$. Hence $z>z_{1}>z_{2}$, by the argument above. Let us derive here some inequalities that will be useful in the following. Since $z<h+3 k$ and $z_{1} \leq z-h$, we conclude $z_{1}<3 k$. Moreover, we know that $z_{2} \geq k$ and $z_{1}>z_{2}$, thus we conclude $z_{1} \geq z_{2}+k \geq 2 k$. Finally, we recall that $h+2 k \leq z<h+3 k$. Now let us look at the relative positions of $\beta$ and $y$.

- $\beta<y$. Then $\beta \leq y-k<2 k$. Since $z_{1} \geq 2 k$, we conclude $\beta<z_{1}$. Thus $\beta \leq z_{1}-h \leq 3 k-h$. We also know that $y_{2} \leq 3 k-h$ because $y_{2}<y \leq y-h$, and because $y<3 k$. Hence, both $\beta$ and $y_{2}$ are contained in the interval $[0 ; 3 k-h$, of width $w<3 k-h$. But $3 k-h \leq h$ by hypothesis, and since $\beta$ and $y_{2}$ must be at least $h$ away, this is impossible.

$-\beta>y$. Then $\beta \geq y+k \geq h+2 k$. This implies that both $\beta$ and $z$ lie in the interval $[h+2 k ; h+3 k[$, of width $w<k$. However, $\beta$ and $z$ must be at least $k$ away from each other, a contradiction.

- $z_{2}>z_{1}$. Hence $z>z_{2}>z_{1}$. In particular, we have $k \leq z_{1}<2 k$. But we also know that $k \leq y_{2}<$ $3 k-h \leq 2 k$. Thus $y_{2}$ and $z_{1}$ both lie in the interval $[k ; 2 k[$, of width $w<k$. But they must be at least $k$ away, a contradiction. 
Altogether, we have shown that every possible case leads to a contradiction. This proves that the initial assumption, $\lambda<h+3 k$, is false. This proves the proposition.

\section{Regular Grids of Degree 4}

\subsection{Upper Bounds}

Proposition $7 \lambda_{h, k}\left(G_{4}\right) \leq h+3 k$ when $h \leq \frac{k}{2}$.

Proof : Consider the $L(1,2)$-labeling depicted in Figure 5(a). This labeling has span 7. If we now substitute labels $0, h, k, h+k, 2 k, h+2 k, 3 k, h+3 k$ to labels $0,1, \ldots, 7$, the new labeling we obtain is an $L(h, k)$-labeling of $G_{4}$. Indeed, it is easy to see that when $h \leq \frac{k}{2}$, each pair of consecutive labels differ by at least $h$, while each other pair of distinct labels differ by at least $k$. Moreover, the largest label used is $h+3 k$, hence the result.
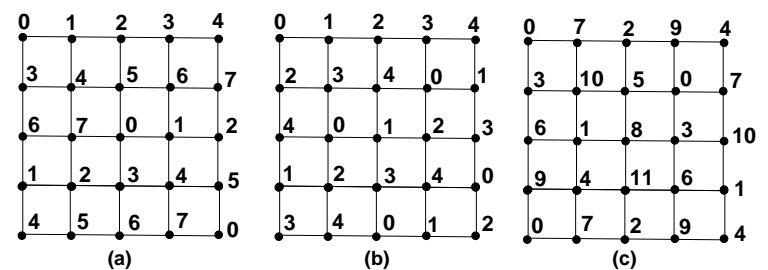

Figure 5: $L(h, k)$-labeling of $G_{4}:$ (a) $L(1,2)$; (b) $L(1,1)$; (c) $L(3,2)$

Proposition $8 \lambda_{h, k}\left(G_{4}\right) \leq \min \{7 h, 4 k\}$ when $\frac{k}{2} \leq h \leq k$.

Proof : By Lemma 4, since $\frac{k}{2} \leq h$ and since there exists an $L(1,2)$-labeling of $G_{4}$ that is of span 7 (as shown in Figure 5(a)), we know there exists an $L(h, k)$-labeling of $G_{4}$ of span $7 h$.

Analogously, since $h \leq k$, we obtain an $L(h, k)$-labeling of span $4 k$ by Lemma 3 ; indeed, there exists an $L(1,1)$ labeling of $G_{4}$ that is of span 4 (as shown in Figure 5(b)).

Proposition $9 \lambda_{h, k}\left(G_{4}\right) \leq 3 h+k$ when $\frac{3}{2} k \leq h \leq \frac{5}{3} k$.

Proof : Consider the $L(3,2)$-labeling of $G_{4}$ depicted in Figure 5(c). This labeling has span 11. If we now substitute labels $0, h-k, k, h, 2 h-k, h+k, 2 h, 3 h-k, 2 h+k, 3 h, 4 h-k, 3 h+k$ to labels $0,1, \ldots, 11$, the new labeling we obtain is an $L(h, k)$-labeling of $G_{4}$. By construction, any pair of labels that are at least 3 away in the list differ by at least $h$, while any pair of labels that is at least 2 away in the list differ by at least $k$, because we supposed $\frac{3}{2} k \leq h$. Moreover, the largest label used is $3 h+k$, hence the result.

Proposition $10 \lambda_{h, k}\left(G_{4}\right) \leq \frac{11}{2} k$ when $\frac{11}{8} k \leq h \leq \frac{3}{2} k$.

Proof : It is known that $\lambda_{h, k}\left(G_{4}\right) \leq 4 h$ when $h \geq k$. Since $\lambda_{h, k}$ is a non decreasing function, Proposition 9 implies that $\lambda_{h, k}\left(G_{4}\right) \leq \frac{11}{2} k$ when $\frac{11}{8} k \leq h \leq \frac{3}{2} k$.

\subsection{Lower Bounds}

Proposition $11 \lambda_{h, k}\left(G_{4}\right) \geq h+3 k$ when $h \leq k$.

Proof: This bound directly comes from Lemma 1. 


\section{Regular Grids of Degree 6}

Proposition $12 \lambda_{h, k}\left(G_{6}\right)=6 k$ when $h \leq k$.

Proof : The upper bound is proved observing that since $h \leq k$, we obtain an $L(h, k)$-labeling of span $6 k$ by Lemma 3 ; indeed, there exists an $L(1,1)$-labeling of $G_{6}$ of span 6 , as shown in Figure 6 . The lower bound directly comes from Lemma 2.

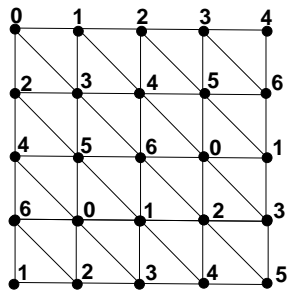

Figure 6: An $L(1,1)$-labeling of $G_{6}$ of span 6

\section{Regular Grids of Degree 8}

\subsection{Upper Bounds}

Proposition $13 \lambda_{h, k}\left(G_{8}\right) \leq 8 k$ when $h \leq k$.

Proof : Since $h \leq k$, we obtain an $L(h, k)$-labeling of span $8 k$ by Lemma 3 ; indeed, there exists an $L(1,1)$ labeling of $G_{8}$ of span 8 (as shown in Figure 7(a)).

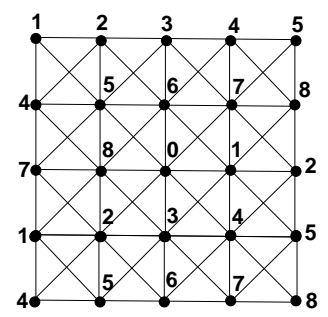

(a)

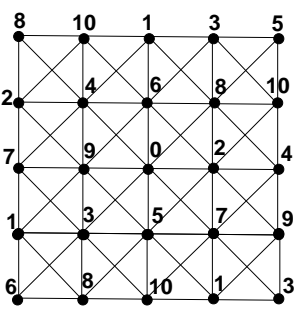

(b)

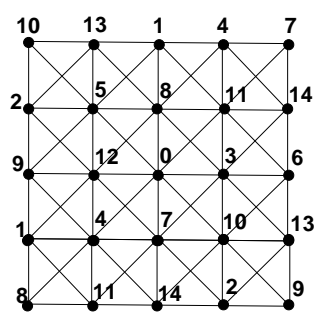

(c)

Figure 7: $L(h, k)$-labeling of $G_{8}:$ (a) $L(1,1)$; (b) $L(2,1)$; (c) $L(3,1)$

Proposition $14 \lambda_{h, k}\left(G_{8}\right) \leq \min \{8 h, 10 k\}$ when $k \leq h \leq 2 k$.

Proof: Once again we exploit the $L(1,1)$-labeling of $G_{8}$ shown in Figure 7 (a). If we substitute $0, h, 2 h, \ldots 8 h$ to labels $0,1, \ldots, 8$, the new labeling we obtain is an $L(h, k)$-labeling of $G_{8}$. Indeed, it is easy to see that each pair of consecutive labels differ by at least $h$, and thus by at least $k$ since $k \leq h$. Moreover, the largest label used is $8 h$, hence the result.

The upper bound of $10 k$ comes from the $L(2,1)$-labeling of $G_{8}$ shown in Figure 7(b). If we substitute $0, k, 2 k, \ldots 10 k$ to labels $0,1, \ldots, 10$, the new labeling we obtain is an $L(h, k)$-labeling of $G_{8}$. Indeed, it is easy to see that when $k \leq h \leq 2 k$, each pair of non consecutive labels differ by at least $2 k \geq h$, while any pair of distinct labels differ by at least $k$. Moreover, the largest label used is $10 k$, hence the result.

Proposition $15 \lambda_{h, k}\left(G_{8}\right) \leq \min \{5 h, 14 k\}$ when $2 k \leq h \leq 3 k$. 
Proof : Consider the $L(2,1)$-labeling described in Figure 7(b). This labeling has span 10. If we now substitute $0, k, h, h+k, 2 h, 2 h+k, 3 h, 3 h+k, 4 h, 4 h+k, 5 h$ to labels $0,1, \ldots, 10$, the new labeling we obtain is an $L(h, k)$ labeling of $G_{8}$. Indeed, it is easy to see that each pair of non consecutive labels differ by at least $h$. On the other hand, since $2 k \leq h$, any pair of distinct labels differ by at least $k$. Moreover, the largest label used is $5 h$.

Analogously, the other bound is given using an $L(3,1)$-labeling, such as the one shown in Figure 7(c). This labeling is of span 14. If we now substitute $0, k, 2 k, \ldots, 14 k$ to labels $0,1, \ldots, 14$, the new labeling we obtain is an $L(h, k)$-labeling of $G_{8}$. Indeed, when $h \leq 3 k$, each pair of labels that are at least 3 away in the list differ by at least $3 k \geq h$, while any pair of distinct labels differ by at least $k$. Moreover, the largest label used is $14 k$, hence the result.

Proposition $16 \lambda_{h, k}\left(G_{8}\right) \leq 4 h+2 k$ when $3 k \leq h \leq 6 k$.

Proof : Starting from the $L(3,1)$-labeling used in the previous proof (cf. also Figure 7(c)) of span 14, we substitute labels $0, k, 2 k, h, h+k, h+2 k, 2 h, 2 h+k, \ldots, 4 h, 4 h+k, 4 h+2 k$ to labels $0,1, \ldots, 14$. This new labeling is also an $L(h, k)$-labeling of $G_{8}$. Indeed, each pair of labels that are at least 3 away in the list differ by at least $h$ by construction, while any pair of distinct labels differ by at least $k$ because $h \geq 3 k$. Moreover, the largest label used is $4 h+2 k$, hence the result.

Proposition $17 \lambda_{h, k}\left(G_{8}\right) \leq 3 h+8 k$ when $h \geq 6 k$.

Proof : Consider the labeling depicted in Figure 8(a). This labeling is an $L(1,1)$-labeling of span 11, with the additional property that the only consecutive labels that can appear on neighboring nodes are of the form $3 i+2$ and $3(i+1)$. We now replace any label $l$ of this labeling by a new label, thanks to the following rule (cf. Figure 8 (b)): any label of the form $l=3 i+j(i=0,1,2,3, j=0,1,2)$ is replaced by $l^{\prime}=(h+2 k) i+j k$. In this new labeling, any pair of labels of the form $3 i+2$ and $3(i+1)$ are now separated by $h$. Moreover, the labeling we started from is an $L(1,1)$-labeling, and any two differing labels in the new labeling are at least $k$ away. Thus, this new labeling is an $L(h, k)$-labeling, of span $3 h+8 k$.

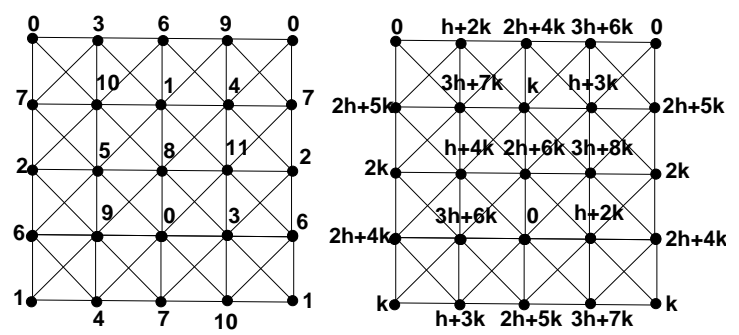

Figure 8: (a) An $L(1,1)$-labeling of $G_{8}$; (b) the $L(h, k)$-labeling we derive

\subsection{Lower Bounds}

Proposition $18 \lambda_{h, k}\left(G_{8}\right) \geq 8 k$ when $h \leq k$.

Proof : This bound directly comes from Lemma 2.

Proposition $19 \lambda_{h, k}\left(G_{8}\right) \geq 2 h+6 k$ when $k \leq h \leq 3 k$.

Proof : Consider any optimal $L(h, k)$-labeling of $G_{8}$. Let $\lambda$ be the greatest label. Let us consider a label $x$ which is neither 0 nor $\lambda$ (note that there must exist one since $G_{8}$ contains $K_{3}$ as an induced subgraph), and consider its 8 neighbors, say $v_{1} \ldots v_{8}$. Then no other label than $x$ can be used in the interval $] x-h ; x+h\left[\right.$ for the $v_{i}$ s. However, all the $v_{i}$ s are pairwise connected by 2-length paths, so they must be at least $k$ away from each other. If there are $\alpha$ (resp. $\beta$ ) labels for the $v_{i}$ s in the interval $[0 ; x-h]$ (resp. $[x+h ; \lambda]$ ), then we must have $(x-h)-(\alpha-1) k \geq 0$ and $\lambda \geq(x+h)+(\beta-1) k$, with $\alpha+\beta=8$. Since $\lambda_{h, k}\left(G_{8}\right)=\lambda$, we conclude that $\lambda_{h, k}\left(G_{8}\right) \geq 2 h+(\alpha+\beta-2) k$, hence the result. 
Proposition $20 \lambda_{h, k}\left(G_{8}\right) \geq 3 h+3 k$ when $h \geq 3 k$.

Proof : First, observe that we have $\lambda_{h, k}\left(G_{8}\right) \geq 3 h+k$. Indeed, consider an optimal $L(h, k)$-labeling of $G_{8}$, a node labeled 0 , and the set of its neighbors (see Figure 9). Wlog, suppose $\min \{a, b, c\} \leq \min \{e, f, g\}$. Since $a$, $b$ and $c$ are neighbors of 0 , then we have $\min \{a, b, c\} \geq h$. And since any node among $f, g$ and $h$ are connected by a 2-length path to any node among $a, b$ and $c$, we conclude that $\min \{e, f, g\} \geq h+k$. Finally, since $e, f$ and $g$ induce a $K_{3}$, we have $\max \{e, f, g\} \geq 3 h+k$.

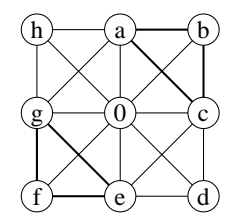

Figure 9: Neighborhood of a node labeled 0 in $G_{8}$.

However, we can derive an even better lower bound, taking into account nodes $d$ and $h$ as well. The result comes from an exhaustive search on the grid restricted to those nine nodes, run by computer (code available at the following URL:

http: //www.sciences.univ-nantes.fr/info/perso/permanents/fertin/Lhk/Lhk.c).

\section{Concluding Remarks}

In this paper, we have studied the $L(h, k)$-labeling problem on regular grids of degree $3,4,6$ and 8 . We observe that the definition we used imposes a condition on labels of nodes connected by a 2-length path instead of using the concept of distance 2, that is very common in the literature. The present formulation (supported by applications) imposes a triangle to be always labeled with three colors at least $\max \{h, k\}$ apart from each other, although its nodes are at mutual distance 1 ; when $h \geq k$, the two definitions coincide.

An open problem arising from this paper consists in closing all the gaps between upper and lower bounds (grey zones in Figure 2).

\section{References}

[1] R. Battiti, A.A. Bertossi and M.A. Bonuccelli. Assigning Codes in Wireless Networks: Bounds and Scaling Properties. Wireless Networks, 5:195-209, 1999.

[2] A.A. Bertossi and M.A. Bonuccelli. Code Assignment for Hidden Terminal Interference Avoidance in Multihop Packet Radio Networks. IEEE/ACM Trans. on Networking, 3:441-449, 1995.

[3] A.A. Bertossi, C.M. Pinotti and R.B. Tan. Channel assignment with separation for interference avoidance in wireless networks. IEEE Transactions on Parallel and Distributed Systems 14:222-235, 2003.

[4] H.L. Bodlaender, T. Kloks, R.B. Tan and J. van Leeuwen. $\lambda$-Coloring of Graphs. In Proc. of STACS 2000. LNCS 1770, 395-406, 2000.

[5] T. Calamoneri. Exact Solution of a Class of Frequency Assignment Problems in Cellular Networks and Other Regular Grids. Proc. 8th Italian Conference on Theoretical Computer Science (ICTCS'03), LNCS 2841, 163-173, 2003.

[6] T. Calamoneri. The $L(h, k)$-Labelling Problem: A Survey. Tech. Rep. 04/2004 Univ. of Rome "La Sapienza", Dept. of Computer Science, 2004.

[7] T. Calamoneri and R. Petreschi. $L(h, 1)$-Labeling Subclasses of Planar Graphs. Journal on Parallel and Distributed Computing 64(3): 414-426, 2004. 
[8] G.J. Chang, W.-T. Ke, D. Kuo, D.D.-F. Liu and R.K. Yeh. On $L(d, 1)$-labelings of graphs. Discrete Mathematics 220:57-66, 2002.

[9] G.J. Chang and D. Kuo. The L(2,1)-labeling Problem on Graphs. SIAM J. Disc. Math., 9:309-316, 1996.

[10] G. Fertin and A. Raspaud. $L(p, q)$ Labeling of $d$-Dimensional Grids. Discrete Applied Mathematics, to appear.

[11] J.R. Griggs and R.K. Yeh. Labeling graphs with a Condition at Distance 2. SIAM J. Disc. Math, 5:586-595, 1992.

[12] W.K. Hale. Frequency assignment: theory and applications. Proc. IEEE, 68:1497-1514, 1980.

[13] P.K. Jha, A. Narayanan, P. Sood, K. Sundaram and V. Sunder. On $L(2,1)$-labeling of the Cartesian product of a cycle and a path. Ars Combin. 55:81-89, 2000.

[14] P.K. Jha. Optimal $L(2,1)$-labeling of Cartesian products of cycles with an application to independent domination. IEEE Trans. Circuits \& Systems I: Fundamental Theory and Appl. 47:1531-1534, 2000.

[15] T. Makansi. Transmitter-Oriented Code Assignment for Multihop Packet Radio. IEEE Trans. on Comm., 35(12):1379-1382, 1987.

[16] B.H. Metzger. Spectrum Management Technique. In Proc. 38th National ORSA Meeting, LNCS 1770, 395406, 1970.

[17] M. Molloy and M.R. Salavatipour. Frequency channel assignment on planar networks. In Proceedings of 10th Annual European Symposium on Algorithms (ESA), LNCS 2461, 736-747, 2002.

[18] D. Sakai. Labeling Chordal Graphs: Distance Two Condition. SIAM J. Disc. Math, 7:133-140, 1994.

[19] M.A. Whittlesey, J.P. Georges and D.W. Mauro. On the $\lambda$ number of $Q_{n}$ and related graphs, SIAM J. Discr. Math. 8 (1995), 499-506. 



\title{
New Bounds for the $L(h, k)$ Number of Regular Grids
}

\author{
Tiziana Calamoneri, Saverio Caminiti, Guillaume Fertin
}

\begin{abstract}
For any non negative real values $h$ and $k$, an $L(h, k)$-labeling of a graph $G=(V, E)$ is a function $L: V \rightarrow \mathbb{R}$ such that $|L(u)-L(v)| \geq h$ if $(u, v) \in E$ and $|L(u)-L(v)| \geq k$ if there exists $w \in V$ such that $(u, w) \in E$ and $(w, v) \in E$. The span of an $L(h, k)$-labeling is the difference between the largest and the smallest value of $L$. We denote by $\lambda_{h, k}(G)$ the smallest real $\lambda$ such that graph $G$ has an $L(h, k)$-labeling of span $\lambda$. The aim of the $L(h, k)$-labeling problem is to satisfy the distance constraints using the minimum span.

In this paper, we study the $L(h, k)$-labeling problem on regular grids of degree $3,4,6$ and 8 , solving several open problems left in the literature.
\end{abstract}

Additional Key Words and Phrases: $L(h, k)$-labeling, triangular grids, hexagonal grids, squared grids, octagonal grids 